\title{
New Developments on Polymer-based MEMS Phase Shifters
}

\author{
Jianqun Wang, Thermpon Ativanichayaphong, Ying Cai, Wen-Ding Huang, \\ Mu Chiao* and J.-C. Chiao \\ Department of Electrical Engineering, University of Texas at Arlington, TX 76019 \\ * Department of Mechanical Engineering, University of British Columbia, Canada
}

\begin{abstract}
Phase shifter is one of the core components for phased-array antennas which find wide applications in satellite systems, telecommunications, wireless systems, radar systems and tracking systems. The current high-frequency phase shifters are mainly built upon active semiconductor technologies which suffer from high fabrication and assembly costs. In this work, we propose a cost-effective approach to fabricate distributed MEMS transmission line phase shifters on a polymer-glass substrate. The fabrication procedure is compatible with CMOS and post-CMOS processes. The polymerglass phase shifter was characterized from DC to $26 \mathrm{GHz}$. The measurements show a phase shift of $120^{\circ}$ and a $2.5-\mathrm{dB}$ insertion loss at $26 \mathrm{GHz}$.
\end{abstract}

\section{INTRODUCTION}

Phase shifters are essential components for many modern communication and radar systems. Conventionally, phase shifters are based on semiconductor or ferrite technologies [1]. Although these technologies proven to be effective, they suffer from some major drawbacks. The high material and fabrication costs, high RF losses and large dimensions increase difficulties for large-array applications. The RF MEMS approach of building phase shifters places a series of distributed MEMS bridges over a coplanar waveguide transmission line (DMTL) [2]. The bridge height is effectively varied by electrostatic forces through biasing MEMS parallel plates. The varied MEMS bridge capacitances slow down the phase velocity and create phase shifts. The DMTL phase shifter has demonstrated higher performance, lower insertion losses, lower power consumption and simpler fabrication processes compared to its semiconductor counterparts [2-3]. However, DMTL phase shifter and its extended approaches are mainly built on quartz [2], intrinsic silicon $[1,4]$ and III-V compound [5] substrates taking advantages of their high resistivity. The material and assembly costs are high.

Comparing to traditional semiconductor materials, polymers are much more cost-effective. Polymers have shown low insertion losses at high frequencies [6-8]. The low dielectric constants, high resistivities and low dissipation factors $[9,10]$ are very attractive for high quality-factor RF applications. Processing polymer does not involve toxic materials nor high thermal budget for annealing. The high thermal stability, high degree of planarization and chemical resistances are compatible with existing IC processing technologies [9,10]. The light weights of polymers are ideally suited for applications where a large number of phase shifters are required. Polymers can also be conformal reducing processing complexity. Many polymers have versatile micromachining abilities which are very attractive for MEMS applications. 3D micro structures can be realized by photolithography, deep reactive ion etching (DRIE), plasma etching [11] or hot embossing processes.

Due to the superb performance of the RF MEMS approach and advantages of using polymer materials, we propose to build distributed MEMS transmission line phase shifters on a polymer substrate. In this paper, design method, fabrication sequences and measurement results will be discussed in details.

MEMS/MOEMS Technologies and Applications III, edited by

Jung-Chih Chiao, Xuyuan Chen, Zhaoying Zhou, Xinxin Li,

Proc. of SPIE Vol. 6836, 68360I, (2007) · 0277-786X/07/\$18 · doi: 10.1117/12.754991

Proc. of SPIE Vol. 6836 68360I-1 


\section{DESIGN CONSIDERATIONS}

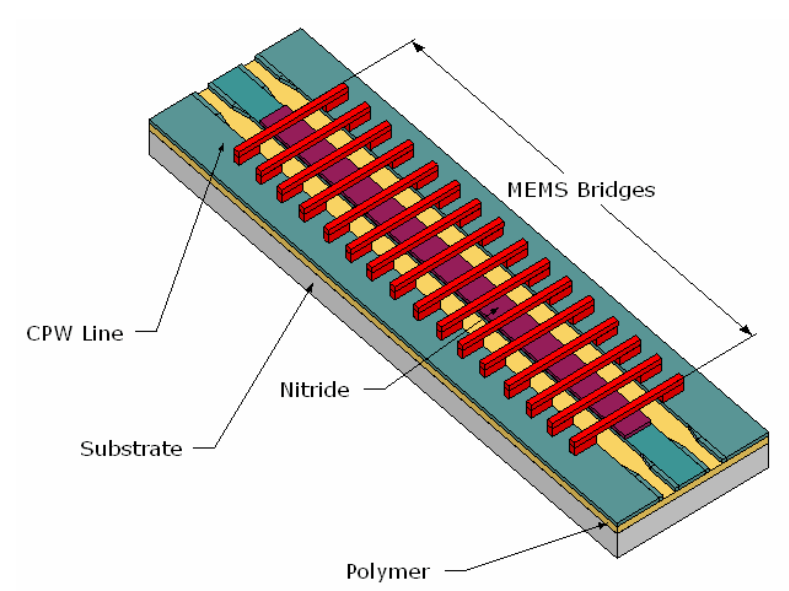

Figure 1. An overall sketch of a RF MEMS phase shifter.

\section{Substrate and Polymer Dielectric Layer}

Unlike intrinsic silicon wafers and III-V compound materials, glass substrates are more cost-effective. Hence, in this work, glass slide is chosen as the substrate material. The polymer will form a layer of insulator to prevent field leakage into the substrate so the resistivity of the glass substrate becomes less important.

Among many polymers, benzocyclobutene (BCB) has a dielectric constant of 2.65 , dissipation factor of $0.08 \%$ and resistivity of $10^{9} \Omega-\mathrm{cm}$. The annealing temperature is $250^{\circ} \mathrm{C}$ which is below most existing IC thermal budgets [9]. Recent years, researches showed that the BCB has very low insertion losses in the applications of coplanar waveguide transmission lines. For example, an insertion loss of $1 \mathrm{~dB} / \mathrm{cm}$ at $40 \mathrm{GHz}$ has been demonstrated [6-8]. Due to these outstanding RF properties, BCB is chosen as the dielectric material. Fig. 1 shows an overall sketch of a DMTL phase shifter where the BCB polymer is applied on top of the glass substrate as the dielectric layer.

\section{Coplanar Waveguide Transmission Line and MEMS Bridges}

The coplanar waveguide (CPW) transmission line is the foundation block of many RF MEMS devices such as switches, varactors and phase shifters. The uniplanar attribute of CPW lines provides convenience for MEMS structure construction and on-wafer characterization [12]. Fig. 2 shows the basic structure of a CPW line and its unit-length equivalent circuit [2], where $L_{t}$, and $C_{t}$ are unit-length inductance and capacitance, respectively.

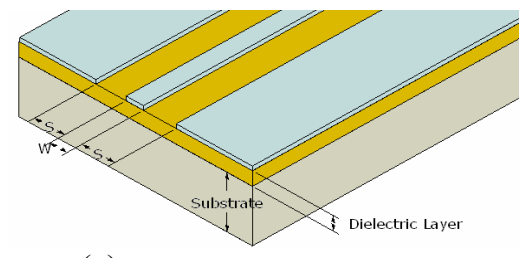

(a)

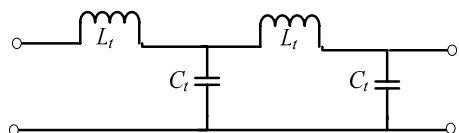

(b)

Figure 2. (a) The CPW line structure. (b) The unit-length equivalent circuit of an unloaded CPW line.

For most RF applications, minimizing the transmission loss is one of the primary goals. Two major factors are the reflection and dissipation losses. To minimize the reflection loss, the characteristic impedance $Z_{o}$ needs to match with that of the rest of the circuitry. In our case, the circuitry network has a characteristic impedance of $50 \Omega$. Eq. 1 shows the relationship between the characteristic impedance $Z_{o}$, unit-length inductance $L_{t}$, capacitance $C_{t}$ and the effective dielectric constant $\varepsilon_{\text {eff }}$ for a CPW line without loading any MEMS bridges. Eq. 2 indicates the phase velocity traveling in a CPW line. 


$$
\begin{aligned}
Z_{o} & =\sqrt{\frac{L_{t}}{C_{t}}} \text { and } C_{t}=\frac{\sqrt{\varepsilon_{\text {eff }}}}{c Z_{o}} \\
V_{u} & =\frac{1}{\sqrt{L_{t} C_{t}}}
\end{aligned}
$$

The dissipation loss consists of three mechanisms: 1) the skin effect, 2) metal conductive losses, and 3) dielectric losses. By properly choosing a good conductor, a micro-scaled MEM device can have very low skin effect and conductive losses. The dielectric loss is inherently low due to the usage of glass substrate and BCB polymer.

The characteristic impedance is decreased when loading a CPW line with MEMS bridges. It adds a shunt capacitance, $C_{b}$, in parallel with the unit-length capacitance, $C_{t}$. Fig. 3 illustrates a CPW line with MEMS bridges loaded and its unitlength equivalent circuit. The capacitance of the loaded CPW transmission line is modeled to increase to $C_{l}=C_{b} / s+C_{t}$ [2], where $s$ is the spacing between two adjacent MEMS bridges. The characteristic impedance of the loaded CPW line is decreased by the $C_{b}$, as shown in Eq. 3 [2]. Therefore, the dimensions of an unloaded CPW line need to be carefully designed so that the characteristic impedance of the loaded CPW line can be close to $50 \Omega$. In our design, the CPW line has a signal width $(W)$ of $100 \mu \mathrm{m}$, signal-ground spacing $(S)$ of $100 \mu \mathrm{m}$, similar to Barker's design in [2]. The unloaded characteristic impedance was calculated to be around $90 \Omega$.

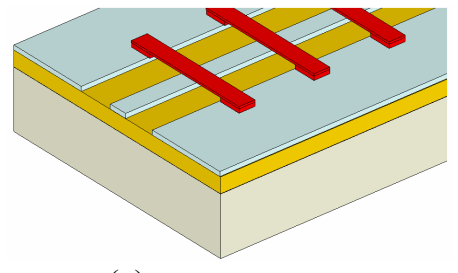

(a)

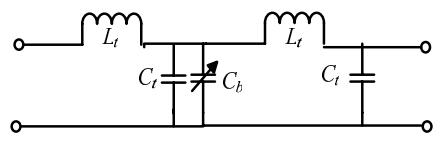

(b)

Figure 3. (a) The loaded CPW line structure. (b) The unit-length equivalent circuit of a loaded CPW line.

$Z_{l}=\sqrt{\frac{L_{t}}{C_{t}+C_{b} / s}}$

The MEMS capacitance slows down the wave velocity, as indicated by Eq. 4 [2]. Thus a phase shift is introduced by the slowed wave velocity. Eq. 5 describes the relationship between the phase shifting value $\delta \varphi$, unloaded phase velocity $V_{u}$ and loaded phase velocity $V_{l}$, where $f$ is the frequency and $l$ is the device length [3].

$$
\begin{aligned}
& V_{l}=\frac{1}{\sqrt{L_{t}\left(C_{t}+C_{b} / s\right)}} \\
& \delta \varphi=2 \pi f l\left(\frac{1}{V_{u}}-\frac{1}{V_{l}}\right) \\
& C_{b}=\varepsilon_{o} \frac{A}{d} \text { and } A=W \times w
\end{aligned}
$$

The capacitance of each MEMS parallel-plate bridge is decided by its dimensions in Eq. 6, where $\varepsilon_{o}$ is the free-space dielectric constant, $A$ is the bridge area overlapped with the CPW signal line, $W$ is the CPW signal line width, $w$ is the bridge width, and $d$ is the height. The parallel-plate capacitance model does not take the edge effects into account. A biasing voltage produces an electrostatic force pulling the MEMS bridges towards to the CPW signal line. Thereby, a larger MEMS capacitance is achieved. In our design, a bridge with a $60-\mu \mathrm{m}$ width is chosen after trading off the elements of phase shifts, losses and characteristic impedance. 


\section{Other Considerations}

The relationship between the biasing voltage $V_{\text {bias }}$ and bridge height $d$ is shown in Eq. 7 [3], where $d_{o}$ is the original bridge height, and $k$ is the spring constant of the bridge membrane which is determined by the material strength, bridge size and surface residue level. A larger original bridge height $d_{o}$ will produce a larger range of MEMS capacitance variation, however a large biasing voltage is needed as well to actuate the parallel plate, as implied by Eq. 7 [3]. A smaller bridge height needs less biasing voltage. Nonetheless, a small bridge height is susceptible to electrical noises and false operations may be introduced. After carefully evaluating these contradicting elements, a bridge height of $0.744 \mu \mathrm{m}$ is designed.

$V_{\text {bias }}=\sqrt{\frac{2 k}{\varepsilon_{o} W w} d^{2}\left(d_{o}-d\right)}$

Only one third of the original bridge height $\left(d_{o}\right)$ can be used to achieve variable capacitances. Beyond the one-third limitation, the MEMS bridges will collapse onto the CPW signal line. This phenomenon is called the "pull-in" condition. The voltage at which the pull-in condition happens is called the "pull-in voltage", as described by Eq. 8 [3].

$V_{p}=\sqrt{\frac{8 k}{27 \varepsilon_{o} W w} d_{o}^{3}}$

The operational frequency is limited by the Bragg frequency, beyond which the CPW line becomes reactive and the return loss increases significantly $[2,13]$. The Bragg frequency is described in Eq. 9.

$f_{\text {Bragg }}=\frac{1}{\pi s \sqrt{L_{t}\left(C_{t}+C_{b} / s\right)}}$

Eqs. 6 and 9 indicate a larger bridge width $(W)$, larger bridge spacing $(s)$ or lower bridge height $(d)$ results in a lower Bragg frequency. In this work, the designed Bragg frequency is above $60 \mathrm{GHz}$, which is more than twice of the upper limit of our measurement ability $(26 \mathrm{GHz})$.

The phase shifter was characterized by two GSG probes which have a $150-\mu \mathrm{m}$ spacing and a $50-\mu \mathrm{m}$ tip width. The phase shifter CPW line consists of a 100- $\mu \mathrm{m}$ spacing and a $100-\mu \mathrm{m}$ signal width. In order to accommodate the dimensional differences and avoid an abrupt impedance change, a taper type matching circuit was designed, as shown in Fig. 4. The insertion loss of such a matching circuit is estimated below $0.15 \mathrm{~dB} / \mathrm{cm}[8,14]$.

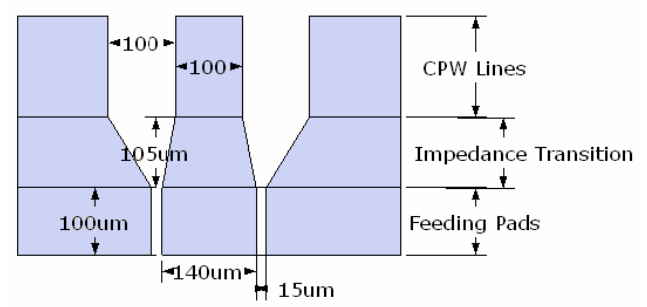

Figure 4. The taper impedance transition. 


\section{FABRICATION PROCESS}

A 10- $\mu \mathrm{m}$ BCB polymer layer was applied on a glass substrate using the spin-on process and inert gas annealing process (Fig. 5(a)). Coplanar waveguide transmission lines were defined on top of the BCB polymer using a sandwiched metal layer of chromium-aluminum-chromium (Fig. 5(b)) with a thickness of 500-8000-500 $\AA$. The two layers of chromium function as an adhesion material. Without such adhesive materials, the aluminum would be easily peeled off in later processes. A silicon nitride layer of $3000 \AA$ was grown on the CPW signal line using the RF sputtering technique (Fig. 5(c)). This silicon nitride layer will prevent MEMS bridges from touching the CPW signal line and resulting in electrical short circuit. A sacrificial layer was applied to define the bridge height using positive photoresist (Fig. 5(d)). A copper layer was deposited using thermal evaporation, followed by photolithography and etching processes to form the MEMS bridge patterns (Fig. 5(e)). In the final step, the devices were soaked into photoresist stripper solution for release (Fig. $5(\mathrm{f})$ ).

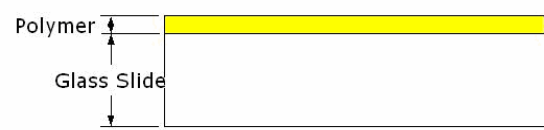

(a)

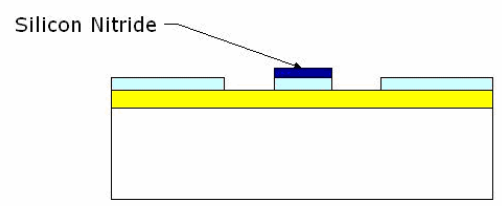

(c)

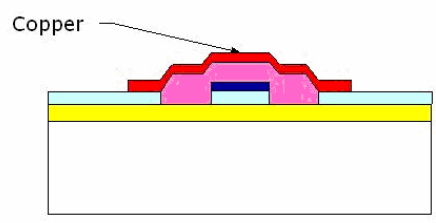

(e)

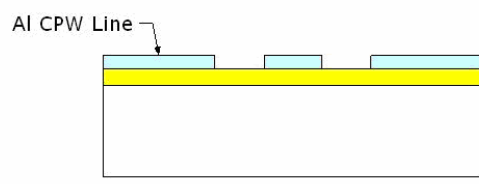

(b)

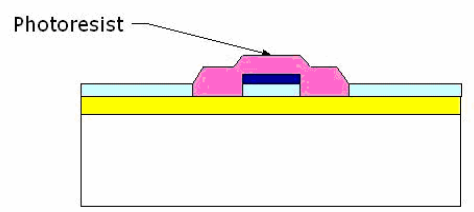

(d)

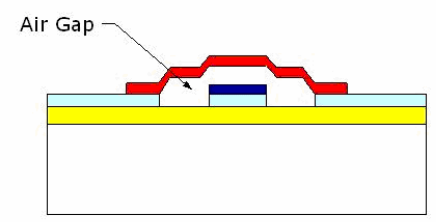

(f)

Figure 5. Fabrication processes. (a) Polymer deposition. (b) CPW transmission line formation. (c) Silicon nitride growth. (d) Sacrificial layer deposition. (e) Bridge formation. (f) Device release.

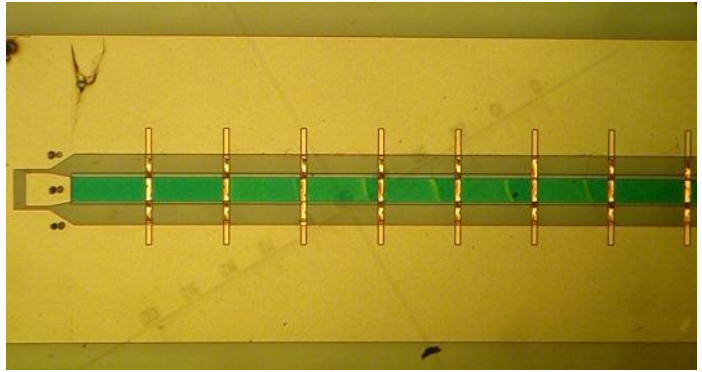

(a)

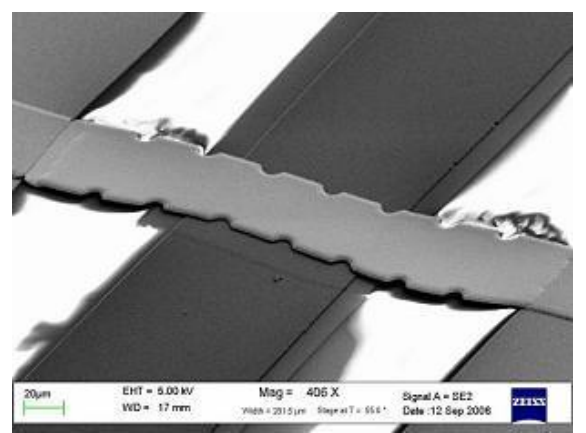

(b)

Figure 6. (a) A microscopic photo of a phase shifter on the polymer dielectric layer. (b) A SEM photo of a MEMS bridge over a CPW signal line. 


\section{EXPERIMENTAL RESULTS}

The DMTL phase shifter was successfully fabricated on a BCB-glass substrate. Fig. 6(a) shows a phase shifter photo under the microscope and Fig. 6(b) shows a SEM picture of a MEMS bridge over the CPW signal line. The dimensions of the RF MEMS phase shifter are shown in Table 1, where $W$ is the CPW signal width, $S$ is the CPW signal-ground spacing, $s$ is the spacing between two adjacent bridges, $w$ is the bridge width, $n$ is the bridge number and $d_{o}$ is the original bridge height.

\begin{tabular}{|c|c|c|c|c|c|c|}
\hline Substrate & $W(\mu \mathrm{m})$ & $S(\mu \mathrm{m})$ & $s(\mu \mathrm{m})$ & $w(\mu \mathrm{m})$ & $n$ & $d_{o}(\mu \mathrm{m})$ \\
\hline BCB-Glass & 100 & 100 & 400 & 60 & 16 & 0.744 \\
\hline
\end{tabular}

Table 1. Phase shifter dimensions.

The device was evaluated by an Agilent 8510 network analyzer and two Cascade Microtech Infinity GSG-150 probes from DC to $26 \mathrm{GHz}$. The scattering parameter measurements were calibrated by a Cascade Microtech ISS-005-016 impedance standard substrate. The DC actuating voltages were applied by an HP4142B DC source through an HP11612 biasing network.

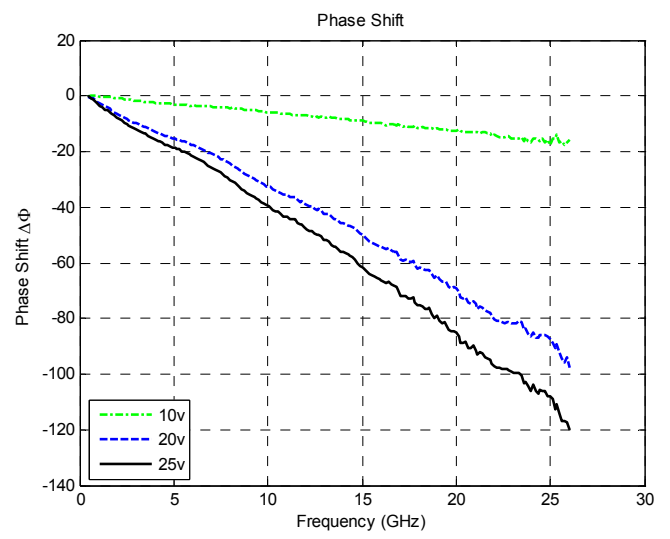

(a)

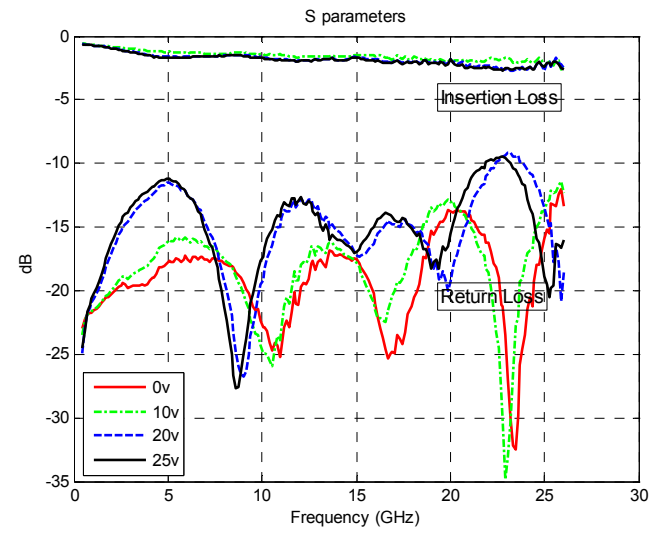

(b)

Figure 7. Experimental results of the phase shifter. (a) Phase shifts versus frequency with different biasing voltages. (b) Insertion and return losses from DC to $26 \mathrm{GHz}$.

Fig. 7(a) shows that at a $10-\mathrm{V}$ biasing voltage, the phase shift reaches $18^{\circ}$ at $26 \mathrm{GHz}$, whereas the phase shift is $95^{\circ}$ at the same frequency at a $20-\mathrm{V}$ biasing voltage. The phase shifts demonstrates a linear increase versus frequency under both biasing voltages. Our testing equipment is limited below $26 \mathrm{GHz}$. This linearity implies that the phase shifting values will keep increasing above $26 \mathrm{GHz}$. Fig. 7(b) shows the scattering coefficients $s_{11}$ and $s_{2 l}$ of the phase shifter at different actuation voltages. The insertion losses are below $2.5 \mathrm{~dB}$ up to $26 \mathrm{GHz}$ regardless of biasing voltages.

The maximum achievable phase shift is limited by the pull-in condition. Under the pull-in condition, the calculated phase shift is $140^{\circ}$ at $26 \mathrm{GHz}$ using Eqs. 4-8. The experimental result shows, with a biasing voltage of $25 \mathrm{~V}$, the bridge height is reduced to $0.5 \mu \mathrm{m}$ which is measured by a Veeco optical profiler. The bridge height reduction is very close to one third of the original bridge height. The phase shift at near the pull-in situation is $120^{\circ}$. The discrepancy may be due to the non-zero surface stress residue of the bridges and the Young's Modulus is higher than the predicted one based on zero-surface stress. The loaded characteristic impedance $Z_{l}$ can be extracted from the first peak of the return-loss spectral curve where the phase shifter behaves as a quarter-wavelength transformer [2]. Using this $Z_{l}$ value, the MEMS bridge capacitance is calculated by Eq. 3. The Bragg frequency is then extracted from Eq. 9. Under this near pull-in condition, the $Z_{l}$ is $38 \Omega, C_{b}$ is $120 \mathrm{fF}$ and the $B r a g g$ frequency is $63 \mathrm{GHz}$, respectively. 


\section{CONCLUSIONS}

In this paper, we investigated the design method, fabrication sequences and measurement results of DMTL phase shifters on polymer substrates. Our measurement indicates that the phase shift reaches $120^{\circ}$ at $26 \mathrm{GHz}$. The insertion loss is less than $2.5 \mathrm{~dB}$. The fabrication processes use low-cost materials and are compatible with CMOS processing.

\section{ACKNOWLEDGEMENT}

This research is sponsored by the National Science Foundation DMI \#0428884. The authors would like to express their gratitude to Dr. N. Scott Barker for his help and valuable suggestions on fabrication, Dr. Ronald Carter for his help on characterization supports, and Dr. Nasir Basit and Mr. Dennis Bueno for their cleanroom assistance.

\section{REFERENCE}

1. T.S. Ji, K.J. Vinoy, and V.K. Varadan, "Distributed MEMS phase shifters by microstereolithography on silicon substrates for microwave and millimeter wave applications," Smart Materials and Structures, vol. 10, pp. 12241229, 2001.

2. N. S. Barker and G. M. Rebeiz, "Distributed MEMS true-time delay phase shifters and wide band switches," IEEE Trans. Microwave Theory Tech., vol. 46, Issue 11, pp. 1881-1890, Nov. 1998.

3. G. M. Rebeiz, "RF MEMS Theory, Design, and Technology,” John Wiley \& Sons, Inc. 2003.

4. Jian Qing, Yanling Shi, Wei Li, Zongsheng Lai, Ziqiang Zhu, and Peisheng Xin, "Ka-band distributed MEMS phase shifters on silicon using AlSi suspended membrane," Journal of Microelectromechanical Systems, Vol, 13, No. 3, June 2004.

5. Rebeiz, G.M., Guan-Leng Tan, and Hayden, J.S., "RF MEMS Phase Shifter: Design and Applications," IEEE Microwave Magazine, Volume 3, Issue 2, pp72-81, June 2002.

6. K. Grenier, V. Lubecke, F. Bouchriha, L. Rabbia. D. Dubuc, P. Pons, and R. Plana, "Polymer in RF and milimeterwave applications," Proc. of SPIE, Volume 5116, pp. 502-513, 2003.

7. L.L.W. Leung, Wai-Cheong Hon and K. J. Chen, "Low-loss coplanar waveguides interconnects on lowresistivity silicon substrate," IEEE Transactions Components and Packaging Technologies, Volume 27, Issue 3, pp.507 - 512, Sept. 2004.

8. Jianqun Wang, Ying Cai, Thermpon Ativanichayaphong, Mu Chiao and J.-C. Chiao, "Fabrication techniques and RF performances of transmission lines on polymer substrates," Proc. of SPIE, Volume 6035, 2006.

9. DOW Processing Procedures For CYCLOTENE 4000 Series Resin.

10. HD MicroSystems PI-2730 Series Low Stress Photodefinable PolyimideProduct Information and Process Guidelines.

11. Sang Won Park, Kabseog Kim, Jeong-Bong Lee, and Wendel Alan Davis, "Plastic-based pattern transfer process for RF MEMS passives," IEEE 2002 Emerging Telecommunication Technologies Conference, Richardson, TX, September 2002.

12. Rainee N. Simons, "Coplanar Waveguide Circuits, Components, and Systems," John Wiley \& Sons, Inc., 2001.

13. M. J. W. Rodwell, S. T. Allen, R. Y. Yu, M. G. Case, U. Bhat tacharya, M. Reddy, E. Carman, M. Kamegawa, Y. Konishi, J. Pusl, and R. Pullela, "Active and nonlinear wave propagationdevices in ultrafast electronics and optoelectronics," Proceedings of the IEEE, vol. 82, no. 7, pp. 1037-1059, July 1994.

14. Janusz Grzyb and Gerhard Tröster, "Characteristic impedance deembeding of printed lines with probe-tip calibrations," Proc. 32nd European Microwave Conference, Milan, Italy, September 23-26, 2002. 